Letras, Lima 48 (82-83): 43-54, 1976.

\title{
Acerca de la distinción entre propiedades y relaciones
}

FERNANDO BOBBIO ROSAS

En este trabajo nos proponemos: a) distinguir, aunque sólo sea de modo preliminar, pero suficiente, entre propiedades (que, en las páginas que siguen, consideraremos sinónimo de cualidades, características, atributos o accídentes), y relaciones; y, b) demostrar que es usual la confusión entre ambos conceptos, en el sentido de considerar las relaciones como si fuesen propiedades. Un tercer punto, que se desprende del anterior y que aquí sólo vamos a rozar, cuando sea imposible evitarlo, es señalar los malentendidos y problemas que origina la mencionada confusión.

La diferencia fundamental entre propiedades y relaciones es la siguiente, las propiedades constituyen integran las cosas; dicho de otra manera, una cosa es el conjunto de sus características ordenadas conforme a una determinada estructura; las características son la cosa, mientras que las relaciones ni son la cosa, ni están en la cosa, sino que son establecidas entre las cosas, (por alguien o por algunos). Como se lee en los textos de lógica:

In ordinary discourse we often speak of relations, which we think of as holding between two things or among several things ( 1 ).

Relations may be... accounted for by identifying them with binary propositional functions, the relation being said to hold between an ordered pair of things (or the things being said to stand in that relation or to bear that relation one to

(1) Patrick Suppes. Introduction to Logic. New York, Van Nostrand Reinhold Co., 1969, p. 210. 
the other) if the binary propositional function is satisfied by the ordered pair (2).

Ahora bien, gramaticalmente, ¿qué términos se refieren a propiedades y cuáles a relaciones?

Consider the proposition 'Aristotle was a Greek'... Grammatically speaking it contains a subject, the word 'Aristotle', which refers to a man, and a predicate, the word 'Greek', which refers to a property of that man. These two parts are not of a similar logical nature but represent the general distinction between a thing and a property... (3).

For the form of the sentence cited, therefore, the symbo. lization $f(x)$ has now been generally accepted. The property is here conceived as a function of the thing, which in turn is regarded as the argument of the function (4).

There are also functions of two variables. For instance, the sentence 'Peter is taller than Paul' refers to a situational function being taller and thus contains the propositional function 'taller'... Accordingly, we shall write the sentence in the form $f(x, y)$.

The two-place situational functions are also called relations. This term, therefore, corresponds to the term 'property' which is usually applied only to one-place situational functions. By predicates we shall understand the names both of properties and of relations (5):

Tenemos, pueș, lquePlac mientionadue alferencia entre propiedades y relaciones en el sentido de que las propiedades están en las cosas, son las cosas, las constituyen, las conforman, las integran, mientras que las relaciones ni están en las cosas, ni son las cosas, sino que son establecidas entre dos o más cosas, tiene su representación, en el simbolismo lógico, en la que se manifiesta entre funciones de un solo argumento, que se usan para repre-

(2) Alonzo Church. Introduction to Mathematical Logic. New Jersey, P. U. P., 1956, vol. I, p. 30, donde además con respecto a 'propiedad, puede leerse lo siguiente:

A property..., differs from a class only or chiefly in that two properties may be different thought the classes determined by them are the same, (where the class determined by a property is the class whose members are the things that have that property). Therefore we identify a property with a class concept or concept of a class...

(3) Hans Reichenbach. Elements of Symbolic Logic. New York, The Free Press, 1966, p. 80.

(4) Op. cit., p. 81 .

(5) Op. cit., p. 83. 
sentar proposiciones compuestas de un sujeto $y$ un predicado: $f(x)$, y funciones de dos o más argumentos: $f(x, y), f(x, y, z)$, etc. En estas fórmulas las cosas se representan con las llamadas variables individuales: $x, y, z, y$ el predicado con la predicativa: f. Y ésta puede referirse tanto a una propiedad de la cosa, como ocurre en el primer caso, o como suponemos que ocurre, en que se trata de la atribución de una propiedad (real o supuesta) a una cosa, como a una relación entre cosas, como sucede, de un modo gráficamente visible, en las dos últimas fórmulas, en las que, claramente, el predicado sirve para relacionar dos y tres cosas entre sí, respectivamente.

Así, establecida la diferencia fundamental que existe entre propiedades y relaciones, podemos pasar al segundo punto de nuestro programa, que constituye la parte central de este trabajo y que, como se recordará, consiste en demostrar que, a menudo, se confunden propiedades y relaciones, tomándose éstas por aquéllas. Dicho de otra manera, vamos a demostrar que muchos términos generalmente considerados como referentes a propiedades, cualidades o características, realmente se refieren a relaciones. Y esta confusión se debe, en buena parte, al hecho de que tanto unas como otras desempeñon el papel de predicados gramaticales, tal como se señala al final de la última de las citas hechas líneas arriba.

Siendo esto así tendemos a clasificar como relaciones exclusivamente aquellos predicados que, para tener sentido, exigen de manera explícita y perentoria más de una cosa, por lo menos dos, entre las cuales se establedéta susodichatrelación. Tal es el caso del ejemplo ofrecido: 'Pedra es imás alto so '; o de innumerables otros ejemplos posibles: 'Pedro es esposo...' ' 'Pedro ama...', 'Pedro recibe...', 'Pedro está entre...', etc. Automáticamente' sentimos que todas estas expresiones carecen de sentido por estar incompletas y, automáticamente también, sentimos la necesidad de completarlas y surgen las preguntas pertinentes: '¿qué?' ¿quién?', '¿de quién?', '¿a quién?', etc. En cada caso la respuesta nos proporciona el otro término, (o los otros términos), de la relación exigido por el predicado y la proposición así completada se llama proposición relacional.

Pero, así como hay casos en los cuales el predicado exige más de una cosa para que la expresión tenga sentido, hay muchos otros casos en los que el predicado gramatical se satisface, (o ésa es la impresión que tenemos), con solamente un sujeto. En todos estos otros casos tendemos a considerar, indiscriminada e irreflexivamente, que dichos predicados se refieren, exclusivamente, a propiedades de la cosa en cuestión, la que hace de sujeto gramatical.

Trataremos de demostrar que no siempre es así y que en muchos casos en los que el predicado, aparentemente, se correspon- 
de con una propiedad, en realidad se refiere a una relación. Consideremos algunos ejemplos:

1. Pedro es alto.

2. Pedro tiene una estatura de dos metros.

3. Aristóteles es griego.

4. Aristóteles es casado.

Todos estos son enunciados que constan de un sujeto gramatical, (una cosa), y un predicado gramatical. Y dicho predicado sólo requiere de una cosa, la que hace de sujeto, para formar una proposición completa y significativa. Todos entendemos lo que quieren decir. A todo esto podemos agregar, y ello es lo más importante para nuestro objetivo, que en los cuatro ejemplos ofrecidos, vistos ligera y superficialmente, tenemos la impresión de que el predicado gramatical consiste en la atribución de alguna propiedad, cualidad o característica al sujeto. Y ésta es la aseveración que ponemos en duda. Examinemos brevemente cada uno de los enunciados anteriores y veamos a qué conclusiones nos conduce el análisis de cada caso.

Tomemos el enunciado del primer ejemplo, 'Pedro es alto'. El sujeto es una cosa, en este caso concreto un ser humano; y el predicado es, o parece ser, una propiedad de dicho ser humano. A Pedro se le atribuye, dice Reichenbach (6), la propiedad de ser alto. Si Pedro posee, real y efectivamente, continúa el autor citado, la mencionada cualidad, el enunciado es verdadero; en caso contrario, el enunciadoes falso.

Que algunas cosas, (seres humanos en el caso del enunciado que estamos examinando), tengan la propiedad, cualidad 0 característica de ser altos, y otros la de ser bajos, es algo generalmente aceptado. Más aún, la diferencia o clasificación que se hace, en los manuales gramaticales, de los adjetivos en calificativos, comparativos y superlativos, contribuye a la suposición de que los primeros expresan, única y exclusivamente, propiedades del sujeto, mientras que de los demás, comparativos y superlativos, sí se admite que sean relacionales, tal como, implícita pero claramente, lo establecen sus propios nombres. Que las categorizaciones gramaticales se nos imponen, en mayor o menor grado, condicionando las imágenes, los conceptos y las teorías que nos forjamos sobre la realidad (7) lo podemos apreciar en la siguien-

(6) Op. cit., p. 82 .

(7) Sobre las relaciones entre el lenguaje, el pensamiento y la realidad o, más claramente, sobre la influencia del lenguaje sobre las imágenes, conceptos y teorías que nos formamos de la realidad existe una bibliografía muy amplia. Dentro de aquellas destacan de un lado, el más filosófico, las obras del último Wittgenstein y sus epígonos oxo- 
te cita, tomada de uno de los textos más completos y rigurosos que se hayan escrito en los últimos años, en la que es claramente visible la influencia de la antedicha clasificación de los adjetivos en calificativos, comparativos y superlativos:

Tomemos, por ejemplo, la altura. Psicológicamente partimos del concepto de clase 'alto'; sobre la base de este concepto introducimos luego el concepto comparativo 'más alto'; por último, construimos el concepto cuantitativo 'altura'. Una vez obtenido este último, volvemos al concepto comparativo y lo cuantificamos (8).

En este pasaje la clase de las cosas altas está considerada como el punto de partida que sirve de base para posteriores comparaciones. Según esto se es alto por derecho propio, es decir, es una clase que se corresponde con una propiedad, la de ser alto $\mathrm{y}$, por lo mismo, integran esta clase todas aquellas cosas que poseen la propiedad de ser altás.

Se parte, se nos dice, del concepto de clase 'alto', el calificativo, correspondiente a una cualidad de las cosas; de aquí se pasa al comparativo 'más alto' que es, claramente ya, un concepto relacional; una cosa puede ser calificada de alta, y generalmente lo es; pero no puede ser calificada, así a secas, de más alta; tiene que serlo de otra u otras, para emplear este concepto se requiere, necesariamente, de dos o más cosas; es imposible aplicarlo, con sentido, a una, sola cosa. El superlativo ni se menciona ni nos es necesario. Blast bufenot least, el concepto cuantitativo 'altura' parece que seoconstruyeiqa partir del 'Somparativo que, a su vez, también se puede cuantificar y, lo más importante, se mantiene, como a todo lo largo de la obra una cuidadosa separación entre conceptos cualitativos y conceptos cuantitativos.

nienses; y de otro, las de Sapir, Whorf y los lingüistas y antropólogos influenciados por estos dos autores. También son interesantes los esfuerzos de Schaff por conciliar estas concepciones, (se refiere abundante y aprobatoriamente a Whorf), con su marxismo.

(8) Mario Bunge. La investigación científica. Barcelona, Ariel, 1969 , p. 765. Que no solamente el hombre común y corriente confunde propiedades y relaciones, tomando éstas por aquéllas, sino que también incurren en el mismo error los epistemólogos, nos lo viene a confirmar, más claramente aún, si cabe, el siguiente pasaje:

No se es, pues, cuñado, como se es alto o bajo; alto lo es uno en sí mismo, mientras que cuñado o abuelo lo es uno solamente con respecto a otros. Tenemos, pues, ideas que poseen un contenido en sí mismas e ideas cuyo contenido es una pura relación a otras. (Roberto Saumells. Fundamentos de matemática y de física. Madrid, Editorial Rialp, 1961, p. 48-49). 
Después de todo lo anterior parecería absurdo dudar de que 'ser alto' sea un concepto relativo a una cualidad. Parecería que, indiscutiblemente, lo es. Las cosas son altas, como son pesadas, duras o buenas (9). Pero, ¿qué sucedería si colocásemos a todos los seres humanos en orden sucesivo de acuerdo a su estatura?

En este caso tendríamos varios miles de millones de variaciones para una diferencia total de altura de no más de siete $u$ ocho pies, lo que da una diferencia promedio entre dos personas contiguas de no más de una cincuentimillonésima parte de una pulgada, diferencia que, naturalmente, no puede apreciarse por los procedimientos corrientes y que es, por cierto, mucho menor que el aumento y disminución normales de altura de una persona ṕor acción del reposo y el ejercicio durante el día. En realidad, si habríamos de colocar en orden de altura a todas las personas del mundo, nos encontraríamos con que los cambios diarios de estatura individual son relativamente mucho mayores que las diferencias medias de estaturas entre los individuos, a tal punto que ese constante cambio obligaría a cambiar de puesto en la línea a cientos de miles y aún a millones de personas, a intervalos relativamente cortos (10).

El punto principal que queda en claro es que entre dos elementos sucesivos de la secuencia no hay ninguna diferencia perceptible y que, más aún, la diferencia que pueda existir entre ambos va a estar bs scilandoincescmtementes ora a favor de uno, ora a favor del otro.

De lo anterior se deduce que es total y absolutamente arbitrario dividir esta secuencia, (o cualquier otra similar), en partes, única manera de obtener las clases correspondientes, en el presente caso, la de los altos y la de los bajos, ya que para ello es necesario trazar una separación entre dos de los elementos de la secuencia que, como se acaba de señalar, de una parte, no son, en ningún sentido de la palabra, perceptiblemente diferentes; y, de otro lado, van a estar cambiando de lugar, constante, incesante $e$ inevitablemente lo que, en el caso de los individuos vecinos a la arbitraria, ilusoria y absurda línea demarcatoria, va a dar lugar a que sean, sucesivamente, altos y bajos en fracciones de tiempo muy pequeñas. Lo cual nos lleva a considerar la antedicha línea separatoria, no sólo como absurda, sino también, como realmente inexistente.

(9) Bunge. Op. cit., p. 159.

(10) Carroll Quigley. La evolución de las civilizaciones. Buenos Aires, Editorial Hermes, 1963, p. 54. 
Evidentemente no faltará alguien que, demostrando tener una mentalidad filosófica, proponga como solución al prob'ema suscitado líneas arriba, (suscitado a quien crea en la existencia de la clase de los altos y de la de los bajos y que trata de mantener su suposición), creando una tercera clase, la de los individuos de talla media, los que no son ni altos ni bajos. Pero, bien mirado el asunto, con esto no se resuelve ningún problema, sino más bien lo que logramos es complicarlo. En efecto, ya no sólo vamos a tener individuos que, por ratos, son altos $y, a$ ratos, bajos, sino que hemos desdoblado el problema $y$, ahora, lo que tenemos es, de un lado, individuos que a ratos son altos y a ratos de talla mediana; $y$, de otro lado, individuos que a ratos son bajos $\mathrm{y}$ a ratos de talla media. Dicho de otra manera, agregando una tercera clase hemos duplicado nuestro problema. Cualquier persona de mediana inteligencia estará de acuerdo con nosotros en el sentido de que agregando clases multiplicaremos el problema y nada más.

Enfoquemos el asunto desde otra perspectiva. Supongamos que todos los seres humanos tuviesen una estatura standard, la que se desee, un metro, dos, tres, etc. Es evidente que todos y cada uno de ellos tendría la propiedad de medir alguna de las magnitudes mencionadas, pero nadie tendría, no tendría sentido afirmar de nadie, la propledad de ser alto, puesto que no hay tal. Lo mismo ocurriría si, hipotéticamente, quedase un solo ser humano sobre la tierra. Tendría una estatura, pero, fuese ésta la que fuese, no sería ni alto ni bajo, excepto, si se quiere, en comparación, en relación, con los que hubo antes; conservaría sus cualidades, pero habría perdido sus relaciones.

Tenemos, pues, que el 'ser alto' es un concepto que no se corresponde con ninguna propiedad de ninguna cosa, sino con una relación que se establece entre los elementos sucesivos de una secuencia dada, (real o imaginaria). Ser alto es ya una relación, una comparación, no un punto de partida para recién hacer luego, en base a él, posteriores comparaciones. Se puede admitir, y de hecho así es, que se parte de un concepto de clase 'alto', pero este es ya un concepto relacional, no cualitativo. 'Ser alto' es una relación, surge de la comparación entre seres de diversas estaturas, de dicha comparación surge la división entre 'altos' Y 'bajos', con todas las complicaciones ulteriores que se quiera, ( $y$ podemos recordar, por ejemplo, la primitiva -en varias de las acepciones de la palabra- clasificación entre pobres y ricos; a la hubo que "enriquecer" con una tercera clase, la clase media, y a las que posteriormente, como ocurre en las amebas y otros animalitos cuando se reproducen, hubo que desdoblar con lo cual tenemos que los sociólogos trabajan al presente, hasta donde llega nuestra información, con seis clases donde originariamente hubo ton sólo dos). 
A todo lo anterior debemos agregar el hecho de que como no existe una comunidad constituida por todos los seres humanos, sino que éstos viven separados en multitud de sociedades, grupos, sub-grupos, micro-grupos, y en las intersecciones entre éstos, la relatividad y subjetividad del concepto 'alto' se complica más aún. Es muy diferente la división que, entre altos y bajos, efectúa un pigmeo, que la que establece un esquimal, un noruego $\circ$ un Watusi. Y así puede ocurrir que si el tal Pedro es un pigmeo y tiene una estatura mayor de un metro y medio es alto, entre los miembros de la comunidad en que vive; mientras que puede muy bien tener una estatura cercana a los dos metros y no ser alto, si es un Watusi.

Lo anterior demuestra, o trata de hacerlo, que nadie es alto, ni bajo a secas, es decir, de manera absoluta, autónoma, independiente, objetiva, sino en relación con otro u otros, dentro de un determinado grupo, entre los miembros de ese grupo, en comparación, implícita o explícita, generalmente implícita, con esos otros. Ser alto, o bajo, no está en el sujeto, no se da en él, no es parte constitutiva de ninguna cosa, como sí lo es, en cambio, el tener una determinada altura o estatura, con todas las precisiones que se quiera y todas las variaciones que se guste tomar en cuenta $a$ lo largo del tiempo que se desee. Luego, no se trata de una propiedad sino de una relación. Las propiedades, como se ha señalado en los comienzos de este trabajo, se dan, están, son la cosa, las relaciones ni se dán, ni están, ni son la cosa, se establecen entre dos o más cosas y esto último es lo que ocurre en el caso examinado, en el cuab como esperamos haberlo demostrado, al afirmar que 'Pedro es alto' cualidad o característica de Pedro, sino una relación que éste guarda con otro o con otros miembros de un grupo determinado y sin la cual no habría dicha relación.

El que 'alto' sea un concepto de relación y no de propiedad, tiene algunas consecuencias interesantes. La primera, y más obvia, es que como tal, es un concepto relativo, (una relación absoluta sería una contradictio in adiecto). Ser alto en Liliput es algo radicalmente diferente a serlo en Brobdignac. Pero no solamente es un concepto relativo, también es un concepto subjetivo, $y$ lo es en grado sumo; depende, en una muy buena parte, del sujeto catalogador, de su estado de ámimo, de sus tendencias, de sus simpatías y antipatías, de su buena o mala voluntad, y varía, o suele hacerlo, con todo ello. Y esta variabilidad es su tercer rasgo importante; es cierto que las propiedades, como todo lo que existe, o mejor, con todo lo que existe, de cuya variación, justamente, ellas son el índice, también varían, pero no en el mismo grado, con la misma rapidez y la misma radicalidad con que pueden variar las relaciones. Un pigmeo 'alto' puesto en algún país 
nórdico se habría convertido en un ser humano 'bajo', bastante bajo. Podemos recordar a Gulliver quien, así de repente, se encuentra convertido en un gigante de los de a de veras, y luego, en un enano en el mejor sentido de la palabra. Mucho más radical, y apenas imaginable, sería la situación si se trata de imaginar al más alto de los liliputienses en el centro de Lorbruldrud, donde ya no sería bajo, ni siquiera enano, sino una especie de microbio. Y el más pequeño de los ciudadanos de Brobdignac sería, recíprocamente, no un ser alto, ni un gigante, sino una especie de bestia apocalíptica si lo trạsladamos a Liliput. La cosa, el pigmeo, Gulliver, el liliputiense, no han variado, en el sentido cotidiano de la expresión, lo que ha variado es el medio y esta variación del medio, es decir, de los otros, determina, a su vez, cambios en la relación o relaciones del sujeto, entre otras, la de su pertenencia a la clase de los altos o bajos. Clases diferentes y relativas, es decir, dependientes, en los diversos grupos en los que se hace la mencionada clasificación. Finalmente, como ya también se ha señalado, la clasificación, el corte, la separación que se hace en la secuencia, linear aquí, para dividir en altos y bajos, (o altos, bajos y de talla media), es total y absolutamente arbitraria. No hay ninguna razón natural u objetiva para colocar la línea divisoria en un lugar determinado y no una, diez, cien o mil unidades antes o después.

Y lo único que se puede afirmar acerca de conceptos relacionales, como los que estamos examinando, con rigurosa objetividad, es que, dentro de una serie o secuencia dada, (de altura en este caso), un elemento cualquiera es, en un determinado momento, más alto que todos losequecestán ubicadossen uno de sus extremos y más bajo que todos aquéllos que están en el opuesto, salvo el primero y el último de la serie que son, respectivamente, el más alto y el más bajo de todos, el alto y el bajo superlativos. Es importante tomar en consideración que la estatura propia de cada miembro de la serie, al determinar el puesto que tiene en ella, determina la relación que guarda con los demás elementos de la misma; lo cual puede ser un factor más que contribuya a la confusión entre propiedades y relaciones, al menos en el presente caso.

Después de todo lo anterior parece que la aseveración del segundo ejemplo está perfectamente clara. En ella se afirma que la cosa representada por el sujeto gramatical, el ser humano llamado Pedro, tiene la propiedad, característica o cualidad de tener una determinada estatura, como todos los seres humanos $y$ que, en su caso concreto es de, (equivalente, igual a), dos metros.

El hecho de tener dos metros, (o dos decímetros o dos decámetros), de estatura es una propiedad de la cosa. Es una propiedad física. Es natural, universal y esencial, en el sentido de 
que es imposible que se carezca de ella, es una consecuencia inevitable de la extensión. Y, además, es perceptible, objetiva, unívoca y absoluta. Esto significa, primero, que todos podemos captar dicha propiedad, en una etapa previa, como una simple relación, con el mismo observador y con los demás miembros del grupo, luego, con mayor exactitud, en relación ya no con los otros, sino con una unidad de medida. Segundo, que es independiente de la apreciación de cualquier sujeto, incluso de quien posee dicha cualidad, (podría ocurrir que alguien creyese tener una estatura diferente a la que en realidad posee). Al ser objetiva, también es unívoca; alto, por ejemplo, al ser un concepto referente a relación, es subjetivo y equívoco: Pedro es alto para unos y bajo para otros, (o puede serlo); alto significa, (o puede significar), que pasa del metro setenta, pero también puede significar que pasa del metro ochenta, o quizá se exija que alguien para ser considerado alto tenga una talla mínima de un metro noventa. Por último, absoluto significa aquí, simplemente, indiscutible, al menos racionalmente; establecido lo que significa tener una determinada estatura, no hay lugar a discrepancia. No se puede establecer, en cambio, al menos racional, y objetivamente, qué significa alto, como lo hemos visto casi hasta la saciedad.

En cuanto al tercer enunciado propuesto, 'Áristóteles es griego', no faltarán quienes piensen que, pese a todo lo dicho anteriormente, aquí también se trata de señalar una propiedad, la de ser griego, de una cosa, Aristóteles. Estamos tan acostumbrados a ello que, automáticamente, lo aceptamos y sostenemos. Pero no es así. 'Ser griego' no es Cuna expresión que se corresponda con ninguna cualidado deeningancelcosanverso"

No hay ninguna cosa que, de por sí, sea 'griega' o 'no-griega'. El término indica, generalmente, la procedencia, el origen de algo y esto, desde luego, es una relación entre, por lo menos dos cosas. Algo más, hay helenos porque hay bárbaros, es una distinción, diferenciación, separación o división entre unos y otros. Hay fieles en tanto que hay infieles $y$, si no hay heterodoxos, tampoco hay ortodoxos.

El 'ser casado' que se predica de Aristóteles en el último ejemplo se refiere también a una relación. Y esto en un doble sentido. Por una parte, en cuanto, como en el caso anterior, el término puede referirse, simplemente, a la división entre casados y solteros $\mathrm{y}$, como acabamos de ver, hay unos en tanto hay los otros, porque hay los otros. Pero, por otra parte, también ocurre que si Aristóteles es casado o, mejor aún, para que Aristóteles pueda ser considerado en el grupo de los casados tiene que estarlo con alguien, tiene que ser marido de alguien, de alguna mujer, o de algunas. Si decimos que 'Aristóteles es marido...', en este caso tenemos, inmediatamente la sensación de que es un enunciado in- 
completo, algo falta; automática e inmediatamente surge la pregunta de rigor: '¿de quién o de quiénes?' Sin embargo, en el enunciado 'Aristóteles es casado', gracias a la construcción gramatical, tenemos la sensación de una oración completa. Pero que ello suceda no significa que 'ser casado' sea una propiedad; no es tal, sino una relación entre, por lo menos, dos cosas, dos seres humanos, (generalmente de sexo diferente), alguien está casado con alguien o no lo está.

Algo más, alguien podría pensar que, a diferencia de lo que ocurre entre altos y bajos, en los casos de los dos últimos ejemplos, la clase de los casados y la de los griegos se distinguen, clara y tajantemente, de las clases de los solteros y de los bárbaros, respectivamente, (y de hecho así podría ocurrir, independientemente de lo que suceda entre altos y bajos), pero no es así. Entre solteros y casados podríamos colocar, cuando menos, a los concubinos. Y si bien el concepto de casado podría ser claro, en cambio el de soltero puede ofrecer problemas. Un hombre de treinta años es, en nuestra sociedad, indiscutiblemente soltero; pero, nos podemos preguntar si tiene sentido predicar tal cosa de un muchacho de quince años; contestaríamos que no, pero podemos recordar que algunas personas ya están casadas a esa edad, ¿entonces?

Un ciudadano ateniense se consideraba a sí mismo y era considerado por todos sus demás conciudadanos heleno; y un persa, del Gran Rey para abajo, era un bárbaro. Pero no faltaron, (ni podían faltar), los 'híbridos', gentes de status dudoso y discutible. Como grupo, los macedonios, dedacépoca de Filipo en adelante, fueron fuente de perplejidad parạlesigriegos』más tradicionalistas, conservadores y reaccionarios que, como tales, creían a rajatabla en divisiones absolutamente excluyentes.

$\mathrm{De}$ todo lo anterior queremos destacar tres puntos que consideramos de suma importancia. El primero es que muchos conceptos aceptados como referentes a propiedades, en realidad se corresponden con relaciones. Y esto ocurre, como hemos visto, no sólo en el caso de lás clases de los altos y de los bajos, sino en muchas otras clases. El segundo es que todos los conceptos referentes a relaciones son arbitrarios, relativos, subjetivos y equívocos. Finalmente, y en relación con lo inmediatamente anterior, se ñalaremos que partiendo del hecho de que las relaciones constituiyen un criterio para la construcción de clases, sin embargo, estas clases no son natural, objetiva ni, mucho menos, radicalmente diferentes entre sí, lo que sí ocurre en el caso de las clases construidas a partir de cualidades de las cosas. Un pato y una gallina son cualitativamente diferentes, pero no lo son un hombre alto y otro bajo, ni tampoco un fiel de un infiel. Sin embargo, diferencias del tipo de esta última han servido, y sirven, para que unos 
seres humanos traten a otros, no como la gallina trata al pato, sino como el zorro trata a ambos, agregando una crueldad que parece ser muy propia del hombre.

Quizá parezca tarea superflua gastar tantas páginas en demostrar, o tratar de hacerlo, todo lo anterior que muchos pueden considerar trivial, pero esto nos recuerda a Swift quien no sólo es el primero en demostrar, con la amplitud necesaria y un buen gusto insuperable, la relatividad y subjetividad de ciertos conceptos, sino que además desnuda, (y al hacerlo nos ridiculiza lo cual, a su vez, nos molesta), algunos de nuestros mecanismos psicológicos, como cuando nos hace el delicioso relato de la guerra, endémica y secular, que sostienen dos bandos de enanos, los unos partidarios de cascar el huevo por la parte ancha, y los otros, de hacerlo por la angosta. El motivo no puede ser más fútil y nos reímos a más no poder de esos pobres infelices. Pero si vamos y vemos un poco más allá y pensamos en la historia y, más grave aún, en el presente de nuestra especie, no lo hallamos tan gracioso. Nos molesta tanto que quizá acudamos al expediente más fácil para desautorizarlo. Lo llamamos misántropo y reaccionario (11). Pero si acudimos al lenguaje para disfrazar la realidad no tendremos ningún derecho a quejarnos de los resultados, mediatos 0 inmediatos.

(11) Lo hace incluso alguien como Orwell, por lo general tan objetivo y mesurado, en un ensayo que le dedica. Cf. Cazando al elefante y otros ensayos. Buenos Aires, Editorial Kraft. 